\title{
ROAD TRANSPORTATION OF DANGEROUS GOODS QUANTITATIVE RISK ASSESSMENT AND ROUTE COMPARISON
}

\section{Philippe CASSINI - INERIS - BP 2 - 60550 Verneuil en Halatte - FRANCE}

Transportation of Dangerous Goods (TDG) by road creates risk for the people present on and along the routes (road users and surrounding population). To minimize them is it better to have TDG going through a city or on the contrary by a longer detour through less populated areas ? The choice is not always easy. It may become very difficult when the detour goes through tunnels. There, accidents may have developments and consequences very different from what might happen on an open air route. To make the right choice it becomes necessary to use a QRA (Quantitative Risk Assessment) approach dealing with the accident scenarios likely to occur, their probability and possible consequences. It may be useful to identify the DG (Dangerous Goods) classes which contribute most to the risk and examine the interest of non-uniform strategies i.e. different routing for certain DG.

\section{Studies for real sites}

INERIS has determined the risks due to TDG for real sites where traffic could go either through a city or an alternative route including a tunnel. Lengths of the tunnels ranked between 2 and $9 \mathrm{~km}$. To perform these studies, INERIS has devised and used a method for determining curves of yearly frequency against number of fatalities corresponding to the risks due to TDG on each route. These curves are hereafter mentioned as « F/N curves ».

This method is very flexible and may be used in very various configurations.

To practically use it, it is necessary to gather, during a first stage, data concerning :

- density of population in the concerned geographical area (possibility to take into account diurnal or seasonal variations),

- traffic of all vehicles and foreseeable routes (possibility to take into account diurnal or seasonal variations),

- traffic of DG : nature of contents and holders, global annual traffic, foreseeable evolutions,

- meteorology in the concerned geographical area,

- layout of the open-air routes (which are divided in «segments » with constant probability of occurrence of each scenario), 
- if it applies, dispositions taken for the design and equipment of the tunnels.

Generally around ten scenarios are chosen (table 1). Five of them are relative to LPGs (propane has been chosen as a representative DG) because of the different physical phenomena they can generate. Their physical consequences (overpressure, heat flux, toxic concentrations for different meteorological conditions) have been calculated and entered in a data-base.

\begin{tabular}{|c|c|}
\hline & Nature of scenario \\
\hline 1 & Explosion of a lorry transporting 15 tons of explosives \\
\hline 2 & $\begin{array}{l}\text { BLEVE (Boiling Liquid Expanding Vapour Explosion) of a } 24 \text { tons vessel of } \\
\text { LPG }\end{array}$ \\
\hline 3 & $\begin{array}{c}\text { VCE (vapor cloud explosion) after a release through a hole of } 5 \mathrm{~cm}^{2} \text { on a } 24 \text { tons } \\
\text { vessel of LPG }\end{array}$ \\
\hline 4 & VCE after a release through a hole of $100 \mathrm{~cm}^{2}$ on a 24 tons vessel of LPG \\
\hline 5 & Jet fire after a release through a hole of $5 \mathrm{~cm}^{2}$ on a 24 tons vessel of LPG \\
\hline 6 & Jet fire after a release through a hole of $100 \mathrm{~cm}^{2}$ on a 24 tons vessel of LPG \\
\hline 7 & VCE after a release through a hole of $80 \mathrm{~cm}^{2}$ on a 30000 litres vessel of gasoline \\
\hline 8 & $\begin{array}{l}\text { Pool fire after a release through a hole of } 80 \mathrm{~cm}^{2} \text { on a } 30000 \text { litres vessel of } \\
\text { gasoline. }\end{array}$ \\
\hline 9 & $\begin{array}{l}\mathrm{NH} 3 \text { dispersion after a release through a hole of } 50 \mathrm{~cm}^{2} \text { on a } 24 \text { tons vessel of } \\
\text { ammonia }\end{array}$ \\
\hline 10 & Fire of lorry transporting 20 tons of NPK fertilizer \\
\hline
\end{tabular}

Table 1 - List of scenarios explored for QRA. 


\section{Determination of the occurrence probabilities and effects}

For each scenario, the zones of effects are determined. For each scenario and each segment of a route, the occurrence probability is determined. These determinations have been backed up by a review of DG accidents occurred during transportation or in industry.

Two numerical tools have been designed to make possible a practical use of the method.

First, a Fortran written program specifically developed at INERIS builds F/N curves for scenarios occuring in the open air. It takes into account a detailed description of population densities and local meteorological conditions. It also indicates everywhere societal and individual risk levels. The individual risk in a given area represents the probability of death due to TDG for one person permanently present there. It is expressed in year ${ }^{-1}$. The local societal risk is affected by population density. In the theoretical case where it would invariably remain equal to one person per $\mathrm{m}^{2}$, both risks are equal. But units are different since societal risk is expressed in year ${ }^{-1} \cdot \mathrm{m}^{-2}$. If there is no one somewhere, local societal risk is naught.

Then a spreadsheet based tool enables to draw $\mathrm{F} / \mathrm{N}$ curves for accidents occurring in tunnels. These accident may produce fatalities inside the tunnels but also outside. Both internal and external contributions are evaluated and summed. In a tunnel, the number of potential victims is linked with densities of trapped road users and their location relatively to the accident. The spreadsheet based tool evaluates this and takes it into account.

Contributions to F/N curves produced by the two tools are summed and F/N curves for routes including open air and tunnel sections produced for every scenario and also for all scenarios together.

Theses studies are very complex. It is therefore impossible to avoid simplifications. The first one being that risk level is evaluated by examination of only a limited number of scenarios corresponding to a very small number of DG. In the same way, collection and prevision of site data are sometimes roughly performed. It is for example difficult to predict 10 or 15 years in advance what will be global traffic, DG traffic and seasonal changes in surrounding population densities.

Validated models for calculation of physical consequences of a few scenarios occurring in tunnel are still missing and work has still to be done in this field where rough assumptions are presently used. The probits (statistical ratio of lethality due to a given exposition) and probability assessment are backed up only by a limited number of studies. 


\section{Minimizing risk between several alternative routes}

In case where DG may be transported by different routes, the method enables to determine the risk that would result from TDG for each route if it were the only one chosen for the DG traffic (DG traffic forbiden on the other routes). Interpretation of results relies on comparing the various $\mathrm{F} / \mathrm{N}$ curves pertaining to the routes or on comparing the various associated «mathematical hopes» (Each F/N curve gives a mathematical hope which represents the area located under the F/N curve. It indicates the yearly number of fatalities when the accidents of every severity are summed up and is expressed in fatalities per year).

This comparison leads to a certain conclusion in the case of $\mathrm{F} / \mathrm{N}$ curves that do not intersect (as shown on figure F1).

It is also possible to get intersecting curves, but with significantly different mathematical hopes (as shown on figure F2), and this even when uncertainties are taken into account. Once more, a conclusion is at hand.

When $\mathrm{F} / \mathrm{N}$ curves intersect and lead to close mathematical hopes, the method does not help in the decision process except give indication that the comparison of risks linked with DG is not discriminating and decision might be made on other criteria.

\section{Judging of the acceptability of risk on a route.}

It is possible to judge of the acceptability of the risks due to TDG on a route by comparing the $\mathrm{F} / \mathrm{N}$ curve with acceptance criteria in case such criteria are available and accepted (Figure 3 shows a fictitious use of such criteria).

Societal risk may also be judged on a local basis using iso-contours like those appearing in Figure 4 . These contours indicate for each area the statistical probability level to get fatalities. These probabilities take into account the population densities and their eventual daily and seasonal variations.

It is also possible to compare individual risk for the surrounding populations with individual risk acceptance criteria.

\section{Conclusion}

QRA of risks due to TDG helps in decision making when routes have to be chosen for DG transit and one wants to minimize resulting risks. It also enables to situate the risks due to TDG against other kind of risks (fatalities due to banal traffic accidents i.e. no 
DG involved for example). It requires complex studies. A method and practical tools have been produced and used for real site cases.

Nevertheless, further work has still to be carried out in order to make these evaluations easier, more accurate and indicate the magnitude of the uncertainties.

\section{List of figures :}

Figure F1 Example of non intersecting F/N curves.

Figure F2 Example of intersecting F/N curves with different mathematical hopes.

Figure 3 Example of comparison of a F/N curve with an acceptance criterion.

Figure 4 Iso-contours of societal risk due to dangerous goods traffic on an open air route (taking into account the population layout).

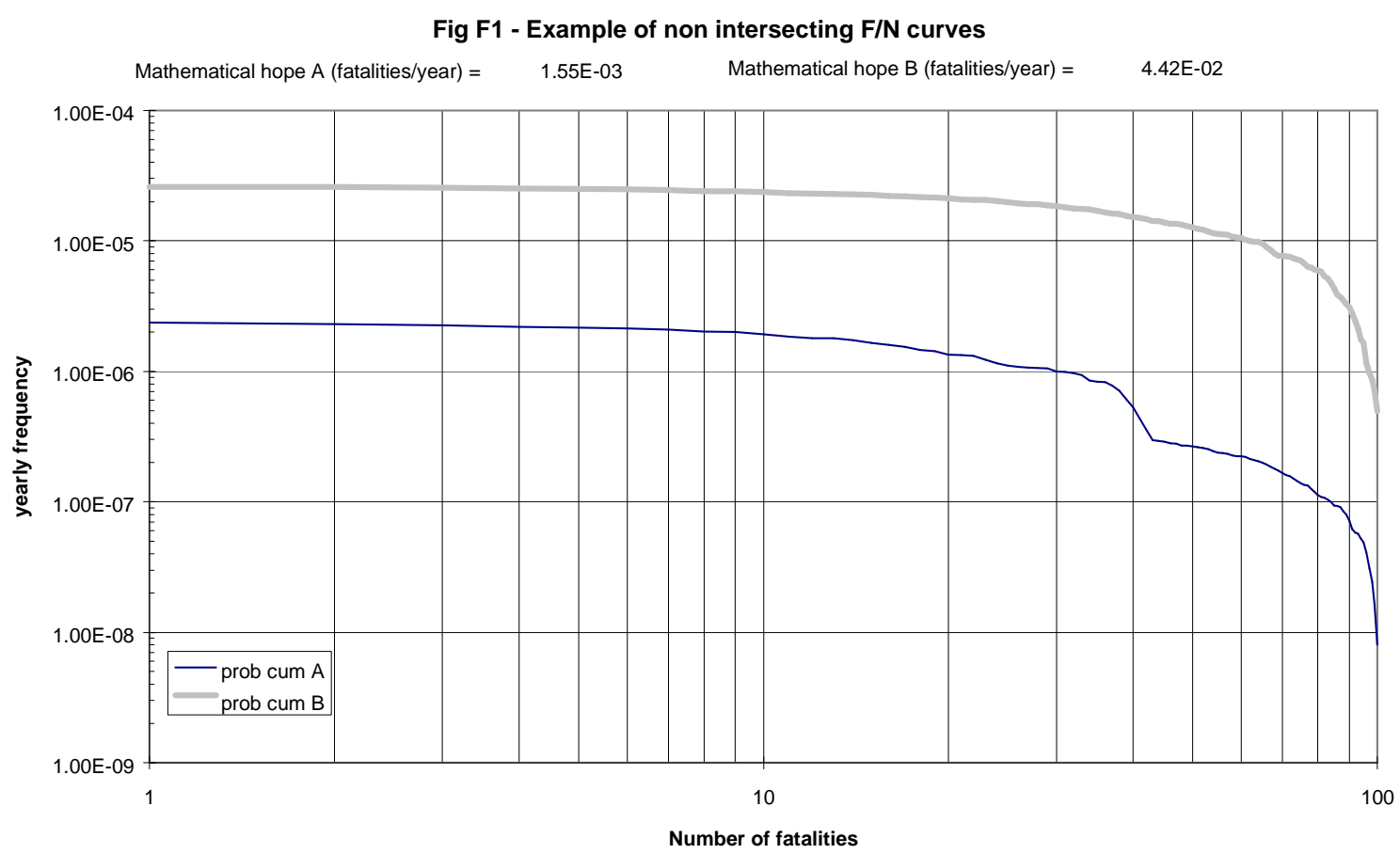


Fig F2 - Example of intersecting F/N curves with very different mathematical hopes

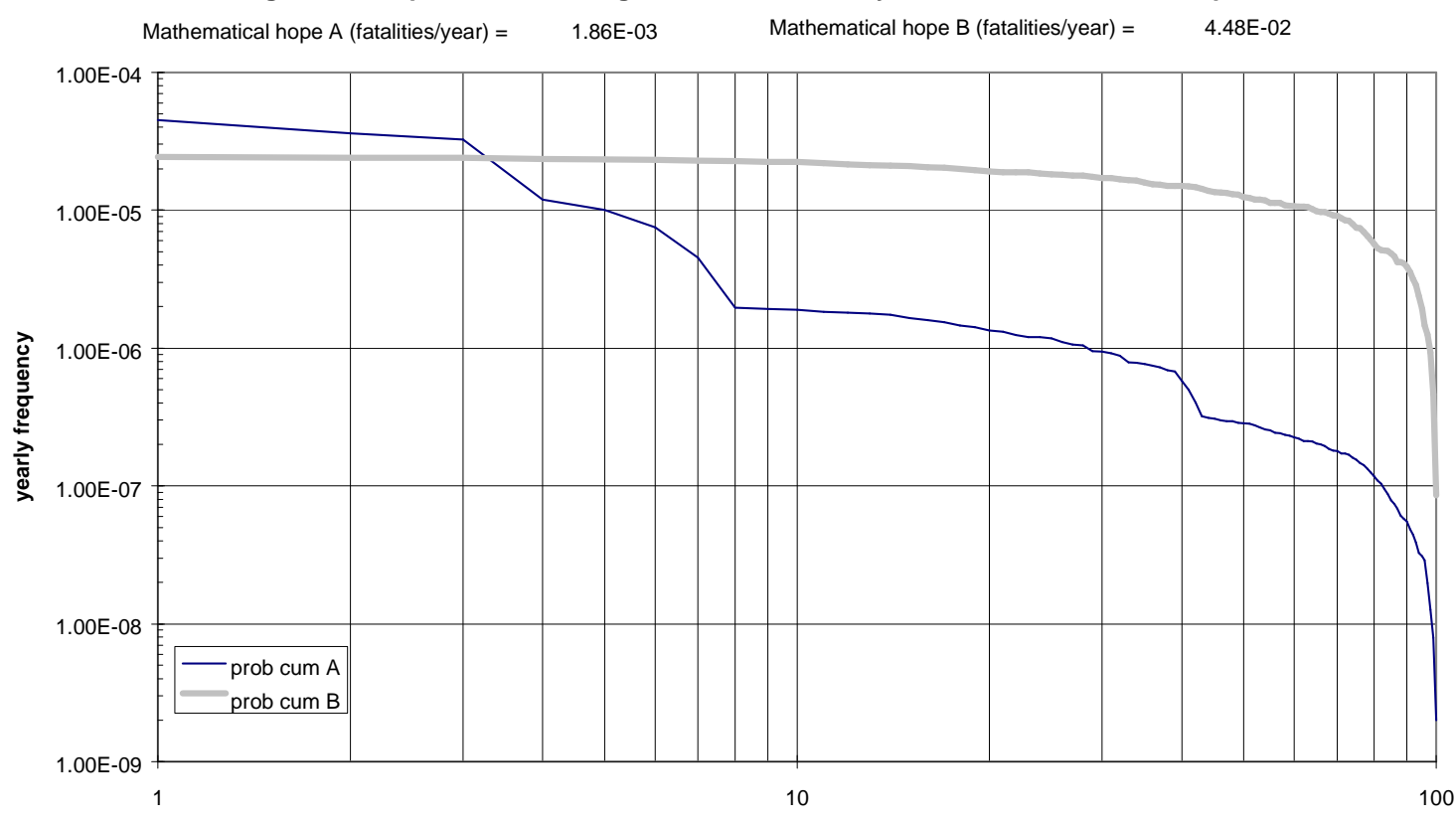

Number of fatalities

Figure 3 : Comparison of a $\mathrm{F} / \mathrm{N}$ curve with an acceptance criteria

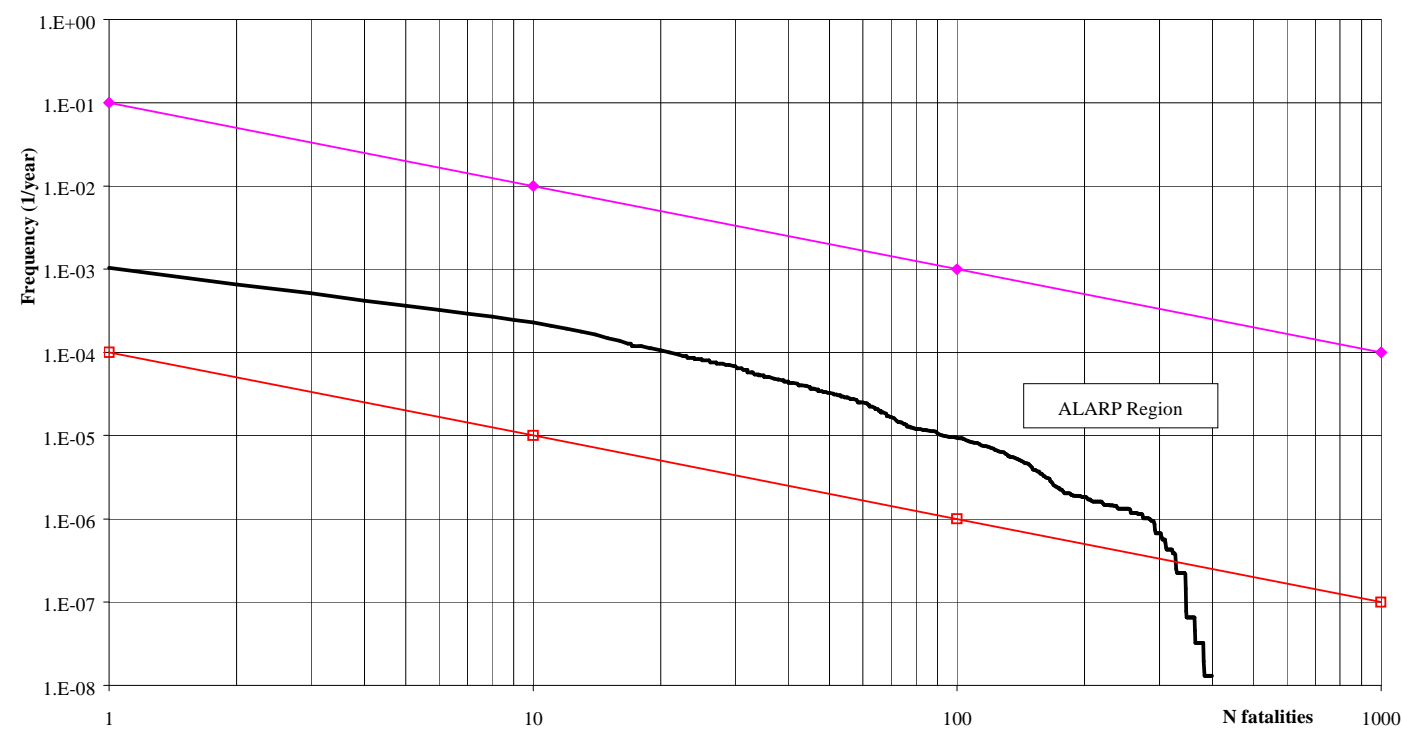


Iso-contours of societal risk due to dangerous goods traffic (taking into account the population layout)

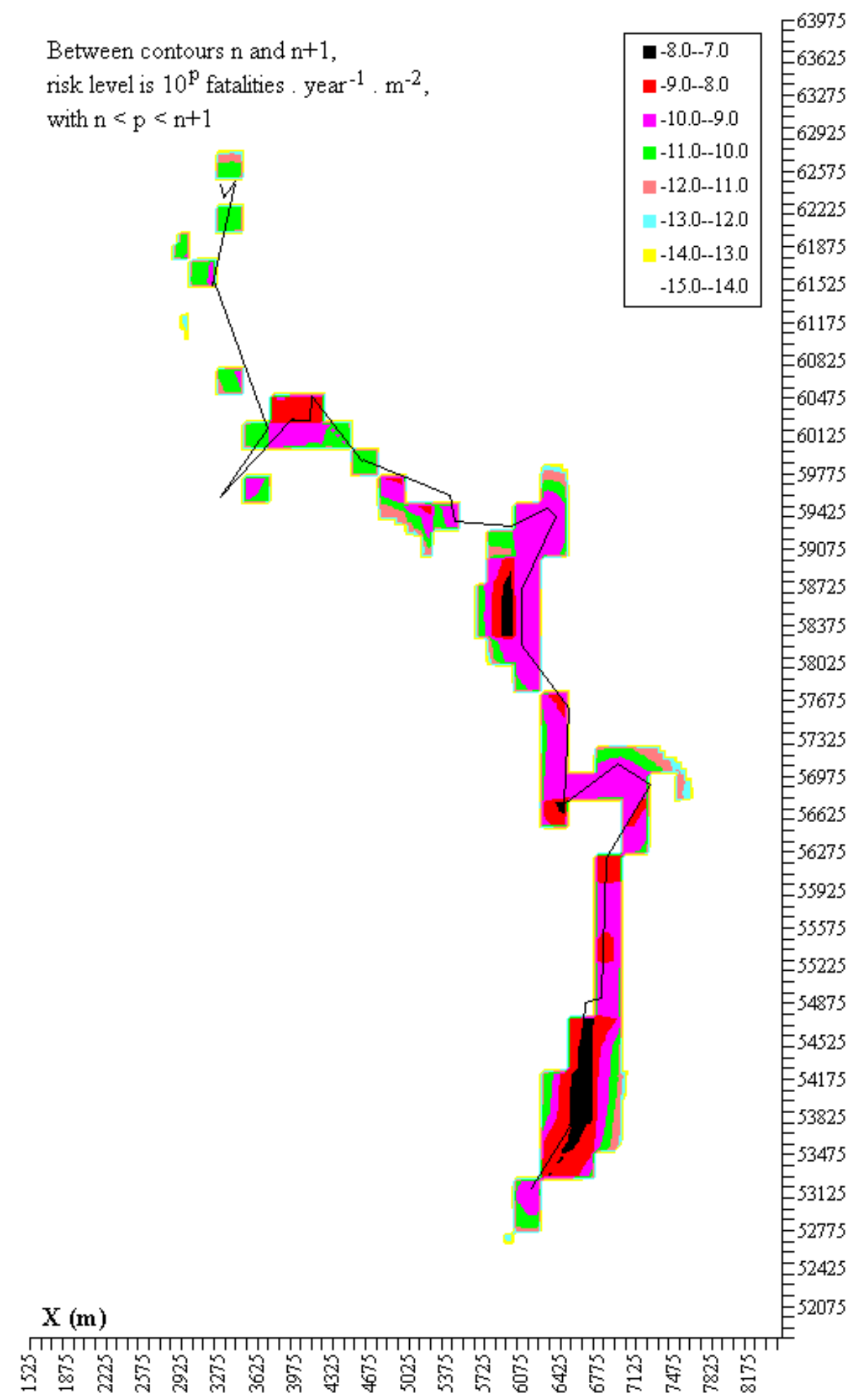

Figure 4

Iso-contours of societal risk due to dangerous goods traffic on an open air route (taking into account the population layout). 